\title{
A influência do exercício físico na qualidade de vida de adultos sobreviventes de câncer
}

\author{
The influence of physical exercise on the quality of life of adult survivors of cancer
}

La influencia del ejercicio físico en la calidad de vida de los sobrevivientes adultos del cáncer

Ana Patrícia de Arruda Silva ${ }^{1 *}$, Themístoclys Thesko Correia Ferreira ${ }^{1}$, Izadora Bezerra Varjão de Melo Santos ${ }^{1}$, Gabriella Carolayne Fernandes Henrique ${ }^{1}$, Thêndallys Yohannãn Correia ${ }^{2}$.

\section{RESUMO}

Objetivo: Analisar na literatura científica a influência do exercício físico na qualidade de vida de adultos sobreviventes de câncer. Métodos: Este estudo trata-se de uma revisão narrativa, realizada nas bases de dados MEDLINE/PUBMED, LILACS, SCIELO e PEDRO, utilizando como critérios de inclusão: ensaios clínicos, com restrição temporal de 5 anos e nas línguas portuguesa, inglesa e espanhola. Os critérios de exclusão foram: estudos que não tivessem a qualidade de vida como objetivo principal e pesquisas que associassem a qualidade vida apenas aos tratamentos farmacológicos. Resultados: Foram analisados sete artigos que descreveram os benefícios do exercício físico na qualidade de vida de sobreviventes de câncer, através de exercícios aeróbicos de baixa e alta dose, treinamento de resistência, atividade física adaptada e exercício para o controle de atenção, resultando assim na redução da dor, melhora do funcionamento físico, fadiga, distúrbios do sono, autoestima, melhora do aspecto psicológico, social e consequentemente o aumento na percepção de qualidade de vida. Considerações finais: $O$ câncer traz graves complicações ao paciente acometido, afetando negativamente a sua qualidade de vida. Há na literatura pesquisas que relacionem a influência do exercício físico na melhora da qualidade de vida dos sobreviventes de câncer.

Palavras-chave: Câncer, exercício físico, Qualidade de vida.

\begin{abstract}
Objective: To analyze in the scientific literature the influence of physical exercise on the quality of life of adult cancer survivors. Methods: This study is a narrative review, performed in the MEDLINE / PUBMED, LILACS, SCIELO and PEDRO databases, using as inclusion criteria: clinical trials, with temporal restriction of 5 years and in Portuguese, English and Spanish. . Exclusion criteria were studies that did not have quality of life as their main objective and studies that associated quality of life with pharmacological treatments only. Results: We analyzed seven articles describing the benefits of physical exercise on the quality of life of cancer survivors, through low and high dose aerobic exercise, resistance training, adapted physical activity and exercise for attention control, thus resulting in pain reduction, improvement of physical functioning, fatigue, sleep disturbance, self-esteem, improvement of the psychological and social aspect and consequently the increase in the perception of quality of life. Final considerations: Cancer brings severe complications to the affected patient, negatively affecting their quality of life. There are studies in the literature that relate the influence of exercise on improving the quality of life of cancer survivors.
\end{abstract}

Key words: Cancer, exercise, Quality of life.

${ }^{1}$ Centro Universitário Brasileiro (UNIBRA). Recife - PE. *E-mail: anapatricia_arruda@hotmail.com

${ }^{2}$ Instituto Paiva. Recife - PE.

SUBMETIDO EM: 8/2019

ACEITO EM: 9/2019

PUBLICADO EM: 10/2019

REAS/EJCH | Vol.Sup.34 | e1501 | DOI: https://doi.org/10.25248/reas.e1501.2019 Página 1 de 8 


\section{RESUMEN}

Objetivo: Analizar en la literatura científica la influencia del ejercicio físico en la calidad de vida de los adultos sobrevivientes de cáncer. Métodos: Este estudio es una revisión narrativa, realizada en las bases de datos MEDLINE / PUBMED, LILACS, SCIELO y PEDRO, utilizando como criterios de inclusión: ensayos clínicos, con restricción temporal de 5 años y en portugués, inglés y español. Los criterios de exclusión fueron: estudios que no tenían como objetivo principal la calidad de vida y estudios que asociaban la calidad de vida solo con tratamientos farmacológicos. Resultados: Analizamos siete artículos que describen los beneficios del ejercicio físico sobre la calidad de vida de los sobrevivientes de cáncer, a través de ejercicios aeróbicos de baja y alta dosis, entrenamiento de resistencia, actividad física adaptada y ejercicio para el control de la atención, lo que resultó en: reducción del dolor, mejora del funcionamiento físico, fatiga, trastornos del sueño, autoestima, mejora del aspecto psicológico y social y, en consecuencia, aumento de la percepción de la calidad de vida. Consideraciones finales: El cáncer trae complicaciones severas al paciente afectado, lo que afecta negativamente su calidad de vida. Existen estudios en la literatura que relacionan la influencia del ejercicio en la mejora de la calidad de vida de los sobrevivientes de cáncer.

Palabras clave: Cáncer, ejercicio, calidad de vida.

\section{INTRODUÇÃO}

O câncer é uma enfermidade crônica, definida pelo desenvolvimento celular desordenado, o que se dá devido a alterações no código genético. Entre $5 \%$ a $10 \%$ das neoplasias são consequência da herança de genes relacionados ao câncer, mas a maioria envolve danos ao material genético, de origem física, química ou biológica, que se aglomeram no decorrer da vida (INUMARU LE, et al., 2011).

Essa doença tem se apresentado como um grande problema de saúde, pois apresenta uma alta prevalência e mortalidade tanto em países desenvolvidos como em países em desenvolvimento. $O$ ordenamento epidemiológico do câncer no Brasil sugere uma transição em andamento, envolvendo um aumento entre os tipos de câncer normalmente associados à alta classe socioeconômica como câncer de mama, próstata e cólon e reto e, concomitantemente presença de taxas de incidência elevadas de tumores geralmente associados com baixa classe socioeconômica como câncer de colo de útero, pênis, estômago e cavidade oral (GUERRA MR, et al., 2015).

Com os avanços para identificação do câncer e o seu respectivo tratamento, observa-se o aumento na sobrevida relativa em cinco anos para em torno de $70 \%$, evidenciando assim uma grande necessidade de desenvolver meios de melhorar a saúde e o bem-estar desses indivíduos. Por se tratar de uma doença sistêmica, a base de tratamento deve incluir recursos que podem afetar a Qualidade de Vida (QV) e a funcionalidade desses pacientes (FRENSHAM LJ, et al., 2018).

Dentre os principais tratamentos clínicos encontra-se a quimioterapia que utiliza medicamentos antineoplásicos para destruir as células tumorais podendo ser curativa, adjuvante, neoadjuvante e paliativa e a radioterapia, que consiste na utilização de feixes ionizantes de radiação para destruir um tumor ou impedir o crescimento celular maligno (LEITE FMC, et al., 2011).

A hormonioterapia é um tratamento através de medicamentos com a função de inibir atividades dos hormônios que tenham alguma influência no crescimento de um tumor. Já a terapia alvo que se utiliza de medicamentos composto de substâncias que foram desenvolvidas para identificar e atacar células cancerígenas, existindo ainda a imunoterapia tendo como objetivo de potencializar o sistema imunológico permitindo combater infecções e outras doenças como câncer ,outra recurso de tratamento muito utilizado no adoecimento oncológico e a cirurgia, que pode ser curativa quando a doença e diagnosticada no estágio inicial (KALIKS RA, et al., 2017).

REAS/EJCH | Vol.Sup.34 | e1501 | DOI: https://doi.org/10.25248/reas.e1501.2019 Página 2 de 8 
Os efeitos colaterais adversos que atingem esta população incluem dor, fadiga, possível comprometimento funcional, morbidade, além de problemas psicológicos, o que afeta negativamente a QV. Estudos mostram que a atividade física pode melhorar a QV em diversos níveis de funcionamento como o físico, emocional, psicológico, social e espiritual. A prática de exercício físico é um meio muito importante para melhorar a qualidade de vida, aptidão física, e problemas relacionados ao sono, como também para reduzir a fadiga em pacientes sobreviventes do câncer (FRENSHAM LJ, et al., 2018, MURTEZANI A, et al., 2014).

Segundo a Organização Mundial de Saúde (OMS) a QV é a percepção do indivíduo de sua posição na vida, no contexto da cultura, sistemas de valores nos quais ele vive em relação aos seus objetivos, expectativas, padrões e preocupações, envolvendo o bem estar físico, mental além de seus relacionamentos com a sociedade e com o meio em que vive, incluindo suas condições de habitação (MAKLUF ASD, et al., 2006).

O exercício físico pode apresentar-se como padrão ouro não só na reabilitação física, mas também no bemestar psicológico e na QV, pois há uma relação entre estilo de vida e sobrevida após o diagnóstico de câncer. A prática de atividade física é uma das principais recomendações para evitar o aparecimento de condições crônico degenerativas. Contudo, estimasse que a inatividade física acomete aproximadamente $75 \%$ da população mundial, sendo mais prevalente entre mulheres e relacionada à classe socioeconômica e raça/etnia (TOLENTINO GP, et al., 2016).

É de suma importância além da prática da atividade física, a reintegração desses pacientes com o meio social na qual geralmente é realizado por meio de participação em grupos e/ou terapias que façam redescobrirem seu corpo e sua capacidade de agir com ele, dessa forma devolve aos pacientes uma melhora na autoestima, no bem-estar e consequentemente na taxa de sobrevida (MOREIRA E MANAIA CAR, 2005).

Diante do exposto, o objetivo deste artigo foi explorar na literatura científica os efeitos do exercício físico na qualidade de vida de adultos sobreviventes de câncer.

\section{MÉTODOS}

O presente estudo trata-se de uma revisão narrativa, realizado nos meses de abril a julho de 2019 , através de pesquisas em artigos científicos publicados com restrição temporal de 5 anos, nas línguas portuguesa, inglesa e espanhola, nas bases de dados: Medical Literature Analysis and Retrieval System Online (MEDLINE/PUBMED), Literatura Latino-Americana e do Caribe em Ciências da Saúde (LILACS), Scientific Electronic Library Online (SCIELO) e Physotherapy Evidence Database (PEDRO).

Para a busca de artigos foram utilizados os Descritores em Ciências da Saúde (DECS): "exercício físico", "câncer" e "qualidade de vida". Para pesquisa na língua inglesa na base de dados MEDLINE/PUBMED e PEDRO foram empregados os termos do Medical Subject Heading (MESH): "physical exercise", "cancer" and "quality of life". Para ampliar as buscas, foi utilizado o Operador Booleano "and".

Quanto aos critérios de elegibilidade, foram incluídos estudos do tipo ensaios clínicos realizados na população humana, na faixa etária adulta. Já os critérios de exclusão adotado nesta revisão foram: estudos que não avaliassem como desfecho principal a Qualidade de Vida e que associassem o exercício físico com terapias adjuvantes.

\section{RESULTADOS}

Para a realização desta revisão foi definida a utilização de estudos originais, que utilizassem na sua metodologia os efeitos do exercício físico na QV de adultos sobreviventes de câncer.

Deste modo, o presente trabalho contou com a análise de 7 artigos, publicados entre 2015 e 2019. As informações contendo as características destes trabalhos (autor/ano de publicação, País da pesquisa, tipo de estudo, amostra, objetivo do estudo, intervenção e resultados), estão descritas em um quadro elaborado pelos autores desta revisão (Quadro 1). 
Quadro 1 - Síntese dos Resultados dos artigos selecionados.

\begin{tabular}{|c|c|c|c|c|}
\hline $\begin{array}{c}\text { Autor/Ano } \\
\text { Tipo de Estudo }\end{array}$ & Amostra & Objetivo & Intervenção & Resultados \\
\hline $\begin{array}{l}\text { Paulo TRS, et al } \\
\text { (2019) } \\
\text { Brasil } \\
\text { Ensaio clínico } \\
\text { randomizado } \\
\text { controlado }\end{array}$ & $\begin{array}{l}\text { Mulheres com idade entre } \\
50 \text { e } 80 \text { anos, com } \\
\text { diagnóstico de câncer de } \\
\text { mama. Foram } \\
\text { randomizados em dois } \\
\text { grupos: treinamento } \\
\text { combinado: resistência } \\
\text { + programa } \\
\text { de exercícios aeróbicos ( } \mathrm{n} \\
=18 \text { ) e grupo controle }(\mathrm{n}= \\
18) \text {. }\end{array}$ & $\begin{array}{l}\text { Avaliar o impacto de } \\
\text { um programa } \\
\text { de exercícios na QV em s } \\
\text { obreviventes de câncer } \\
\text { de mama. }\end{array}$ & $\begin{array}{l}\text { A QV foi avaliada pelo SF36, EORTC } \\
\text { QLQ-C30 e EORTC QLQ- } \\
\text { BR23. O grupo de exercícios realizou } \\
40 \text { min de exercícios de resistência } \\
\text { em máquinas, seguidos de } 30 \text { min de } \\
\text { treinamento aeróbico em esteira } \\
\text { rolante 3x semana. }\end{array}$ & $\begin{array}{l}\text { Interações significativas de tempo x } \\
\text { grupo e tamanhos de efeito moderado } \\
\text { a alto foram encontrados } \\
\text { para funcionamento físico, } \\
\text { saúde física, dor corporal, percepção } \\
\text { geral de saúde, vitalidade, função } \\
\text { social, fadiga, distúrbios do sono e } \\
\text { imagem corporal, favorecendo o grupo } \\
\text { de exercícios. }\end{array}$ \\
\hline $\begin{array}{l}\text { Brown JC, et al. } \\
(2018) \\
\text { EUA } \\
\text { Ensaio } \\
\text { randomizado } \\
\text { dose-resposta } \\
\text { Ensaio } \\
\text { randomizado } \\
\text { dose-resposta }\end{array}$ & $\begin{array}{l}39 \text { Pacientes com Câncer } \\
\text { de cólon de estágio I a III } \\
\text { foram alocados } \\
\text { aleatoriamente em um dos } \\
\text { três grupos: controle de } \\
\text { cuidados habituais ( } \mathrm{n}: 13 \\
\text { participantes), exercícios } \\
\text { aeróbicos de baixa dose ( } \mathrm{n} \text { : } \\
14 \text { participantes) ou } \\
\text { exercícios aeróbicos de } \\
\text { alta dose (n: } 12 \\
\text { participantes). }\end{array}$ & $\begin{array}{l}\text { Examinar os efeitos } \\
\text { dose-resposta } \\
\text { do exercício aeróbico na } \\
\text { QVRS } \\
\text { entre sobreviventes } \\
\text { de câncer de cólon. }\end{array}$ & $\begin{array}{l}\text { O exercício aeróbico foi realizado } \\
\text { durante seis meses utilizando } \\
\text { esteiras domiciliares. Os grupos de } \\
\text { dose baixa e de alta dosagem } \\
\text { progrediram no sentido do objetivo de } \\
150 \text { ou } 300 \text { min. A QVRS específica } \\
\text { do câncer de cólon foi quantificada } \\
\text { utilizando-se a FACT-C. }\end{array}$ & $\begin{array}{l}\text { Durante } 6 \text { meses, o exercício } \\
\text { melhorou o escore do componente } \\
\text { físico do SF-36 ( } p=0,002) \text {, a Avaliação } \\
\text { Funcional da Terapia do Câncer- } \\
\text { Colorretal ( } p=0,025) \text {, o Índice de } \\
\text { Qualidade do Sono de Pittsburgh } \\
(p=0,049) \text { e a Fadiga }(p=0,045) \text {. }\end{array}$ \\
\hline $\begin{array}{l}\text { Landry S, et al. } \\
(2018) \\
\text { França } \\
\text { Ensaio clínico } \\
\text { controlado } \\
\text { randomizado }\end{array}$ & $\begin{array}{l}23 \text { Mulheres } \\
\text { diagnosticadas com câncer } \\
\text { de mama. } \\
\text { O grupo experimental ( } \mathrm{n} \text { : } \\
\text { 13) praticou AFA por } 12 \\
\text { semanas, enquanto o } \\
\text { grupo controle ( } \mathrm{n} \text { : 10) não } \\
\text { o fez. }\end{array}$ & $\begin{array}{l}\text { Avaliar a influência de um } \\
\text { programa de AFA na } \\
\text { autoestima e qualidade } \\
\text { de vida em pacientes } \\
\text { com câncer de mama. }\end{array}$ & $\begin{array}{l}\text { O programa AFA consistiu em } 1 \\
\text { sessão por } 12 \text { semanas, com } \\
\text { duração de } 1 \text { hora. Com exercícios } \\
\text { de fortalecimento muscular, equilíbrio } \\
\text { e flexibilidade. A QV foi avaliada pelo } \\
\text { EORTC QLQ-C30, e pelo EORTC } \\
\text { QLQ-BR23. }\end{array}$ & $\begin{array}{l}\text { Os resultados mostram que a AFA é } \\
\text { benéfica para ambos os parâmetros. } \\
\text { Autoestima, percepção física, QV, } \\
\text { estado global de saúde, dor e } \\
\text { sintomas mamários foram melhorados } \\
\text { apenas no grupo que praticava } \\
\text { atividade física adaptada. }\end{array}$ \\
\hline $\begin{array}{l}\text { Zhou Y, et al. } \\
\text { (2017) } \\
\text { EUA } \\
\text { Ensaio clínico } \\
\text { controlado } \\
\text { randomizado }\end{array}$ & $\begin{array}{l}\text { Sobreviventes de câncer } \\
\text { de ovário, um total de } 74 \\
\text { mulheres foram } \\
\text { aleatoriamente designadas } \\
\text { para o exercício e } 70 \text { para } \\
\text { o controle de atenção. }\end{array}$ & $\begin{array}{l}\text { Examinar o efeito de uma } \\
\text { intervenção de exercícios } \\
\text { aeróbicos de seis meses } \\
\text { contra o controle de } \\
\text { atenção sobre a } \\
\text { mudança na QVRS e } \\
\text { fadiga relacionada ao } \\
\text { câncer. }\end{array}$ & $\begin{array}{l}\text { As mulheres no braço de exercício } \\
\text { participaram de } 150 \text { minutos por } \\
\text { semana de exercícios aeróbicos de } \\
\text { intensidade moderada, } \\
\text { principalmente caminhada rápida. }\end{array}$ & $\begin{array}{l}\text { A mudança na QVRS física foi } \\
\text { significativamente maior no grupo que } \\
\text { exercitou } 150 \text { ou mais minutos por } \\
\text { semana }(p=0,01) \text {, assim como } \\
\text { a mudança na escala FACT-F ( } p= \\
0,02) \text {. }\end{array}$ \\
\hline
\end{tabular}

REAS/EJCH | Vol.Sup.34 | e1501 | DOI: https://doi.org/10.25248/reas.e1501.2019 Página 4 de 8 


\begin{tabular}{|c|c|c|c|c|}
\hline $\begin{array}{l}\text { Shobeiri F, et al. } \\
(2016) \\
\text { Irã } \\
\text { Ensaio clínico } \\
\text { controlado } \\
\text { randomizado }\end{array}$ & $\begin{array}{l}\text { Mulheres com câncer de } \\
\text { mama, sem evidência de } \\
\text { metástase e uso atual de } \\
\text { terapia hormonal. Foram } \\
\text { randomizados para o grupo } \\
\text { de exercícios ( } n=30 \text { ) ou } \\
\text { grupo de controle }(n=30) \text {. } \\
\end{array}$ & $\begin{array}{l}\text { Avaliar o papel do } \\
\text { exercício aeróbico na QV } \\
\text { de mulheres em } \\
\text { tratamento para o câncer } \\
\text { de mama. }\end{array}$ & $\begin{array}{l}\text { O grupo de exercícios recebeu } \\
\text { exercício supervisionado } 2 \text { dias por } \\
\text { semana por } 40-60 \text { minutos por } 10 \\
\text { semanas. Os exercícios incluíam: } \\
\text { aquecimento, exercícios aeróbicos e } \\
\text { relaxamento. A QV foi avaliada pelo } \\
\text { EORTC QLQ-C30. }\end{array}$ & $\begin{array}{l}\text { A QV global do estado de saúde } \\
\text { evoluiu significativamente no grupo } \\
\text { exercício }(p<0,001) \text {. A intervenção do } \\
\text { exercício foi associada com um } \\
\text { desenvolvimento substancial no } \\
\text { escore total de funções e sintomas de } \\
\text { QV }(p<0,001) \text {. }\end{array}$ \\
\hline $\begin{array}{l}\text { Hagstrom AD, et } \\
\text { al. (2015) } \\
\text { Austrália } \\
\text { Ensaio Clínico } \\
\text { controlado } \\
\text { randomizado }\end{array}$ & $\begin{array}{l}\text { Foram recrutadas } 39 \\
\text { mulheres em tratamento } \\
\text { para o câncer de mama, } \\
\text { com idade entre } 18 \text { e } 70 \\
\text { anos. } \\
\text { O grupo intervenção tinha } \\
20 \text { participantes e o grupo } \\
\text { controle } 19 .\end{array}$ & $\begin{array}{l}\text { Avaliar os benefícios do } \\
\text { treinamento resistido na } \\
\text { QV e fadiga em } \\
\text { sobreviventes de câncer } \\
\text { de mama como adjuvante } \\
\text { ao tratamento usual. }\end{array}$ & $\begin{array}{l}\text { Programa supervisionado de } \\
\text { treinamento resistido de } 16 \text { semanas, } \\
\text { 3x por semana durante } \\
\text { aproximadamente } 60 \text { min por sessão. } \\
\text { Três séries de oito a dez repetições } \\
\text { foram realizadas em cada exercício. } \\
\text { A QV foi avaliada pelo FACT-G da } \\
\text { QV. }\end{array}$ & $\begin{array}{l}\text { Houve melhora na fadiga e força no } \\
\text { membro tratado e mudanças na QV e } \\
\text { força relacionada entre si no grupo } \\
\text { TR, mas não no grupo controle. Houve } \\
\text { uma correlação significativa entre } \\
\text { melhorias na fadiga e melhorias na QV } \\
\text { geral no grupo de exercício ( } p= \\
0,004 \text { ). }\end{array}$ \\
\hline $\begin{array}{l}\text { Filha JGLC, et al. } \\
(2016) \\
\text { Brasil } \\
\text { Ensaio clinico }\end{array}$ & $\begin{array}{l}24 \text { mulheres com } \\
\text { diagnóstico de câncer de } \\
\text { mama, na faixa de } 40 \text { a } 67 \\
\text { anos, em continuidade com } \\
\text { tratamento adjuvante. } \\
\text { GE }(\mathrm{N}=12) \text {, submetido a } \\
\text { exercícios e } \mathrm{GC}(\mathrm{N}=12), \\
\text { não submetido. }\end{array}$ & $\begin{array}{l}\text { Investigar a relação entre } \\
\text { o exercício físico e seus } \\
\text { efeitos na QV em } \\
\text { pacientes com câncer de } \\
\text { mama, pós-cirurgia ( } 6 \\
\text { meses). }\end{array}$ & $\begin{array}{l}\text { O programa de exercícios ao GE } \\
\text { durou } 10 \text { semanas, } 3 \text { x por semana, } \\
\text { durante } 50 \text { minutos, com } \\
\text { intensidades submáximas. } \\
\text { Realizados exercícios de resistência } \\
\text { aeróbia e alongamentos gerais. A QV } \\
\text { foi mensurada pelo SF-36. }\end{array}$ & $\begin{array}{l}\text { O estudo mostrou que para o GE em } \\
\text { relação ao GC, houve melhorias nas } \\
\text { áreas: Vitalidade, funcionamento } \\
\text { social e limitações-emocionais. } \\
\text { exercício físico durante o tratamento } \\
\text { do câncer ajuda na melhoria } \\
\text { psicológica, social e física. }\end{array}$ \\
\hline
\end{tabular}

Fonte: Silva APR, et al. (2019).

Legenda: AFA: atividade física adaptada; EORTC QLQ-BR23: Scale of the European Organization for Breast Cancer Research and treatment (Escala da Organização Europeia para Pesquisa e Tratamento do câncer de mama); EORTC QLQ-C30: Scale of the European Organization for Cancer Research and treatment, titled "Quality of Life Questionnaire version 3.0 (Escala da Organização Europeia para Pesquisa e Tratamento do Câncer, intitulado "Questionário de Qualidade de Vida versão 3.0); EUA: United States of America (Estados Unidos da América); FACT-C: Functional evaluation of cancer therapy-Coloretal (Avaliação Funcional da Terapia do Câncer-Coloretal); FACT-F: Functional evaluation of cancer-fatigue therapy (Avaliação Funcional do Terapia do Câncer-Fadiga); FACT-G: Functional evaluation of cancer-fatigue therapy (Avaliação Funcional da Terapia do Câncer-Geral); FC: Frquência cardíaca; GC: Grupo Controle. GE: Grupo Experimental. IC: Intervalo de confiança; MIN: minutos; N: número de participantes; p: nível de significância estatística; QV: Qualidade de vida; QVRS: Qualidade de vida relacionada a saúde; SF-36: Medical Out comes Study 36 - Item Short - Form Health Survey (Estudo de resultados médicos 36 - item Short - pesquisa de saúde de formulário). TR: Treinamento Resistido.

REAS/EJCH | Vol.Sup.34 | e1501 | DOI: https://doi.org/10.25248/reas.e1501.2019 Página 5 de 8 
A seleção dos estudos identificados pela busca foi realizada segundo os critérios de elegibilidade proposto pelo fluxograma desenvolvido pelo the PRISMA statement (Figura 1).

Figura 1 - Busca e seleção dos artigos para a revisão de literatura de acordo com o PRISMA.
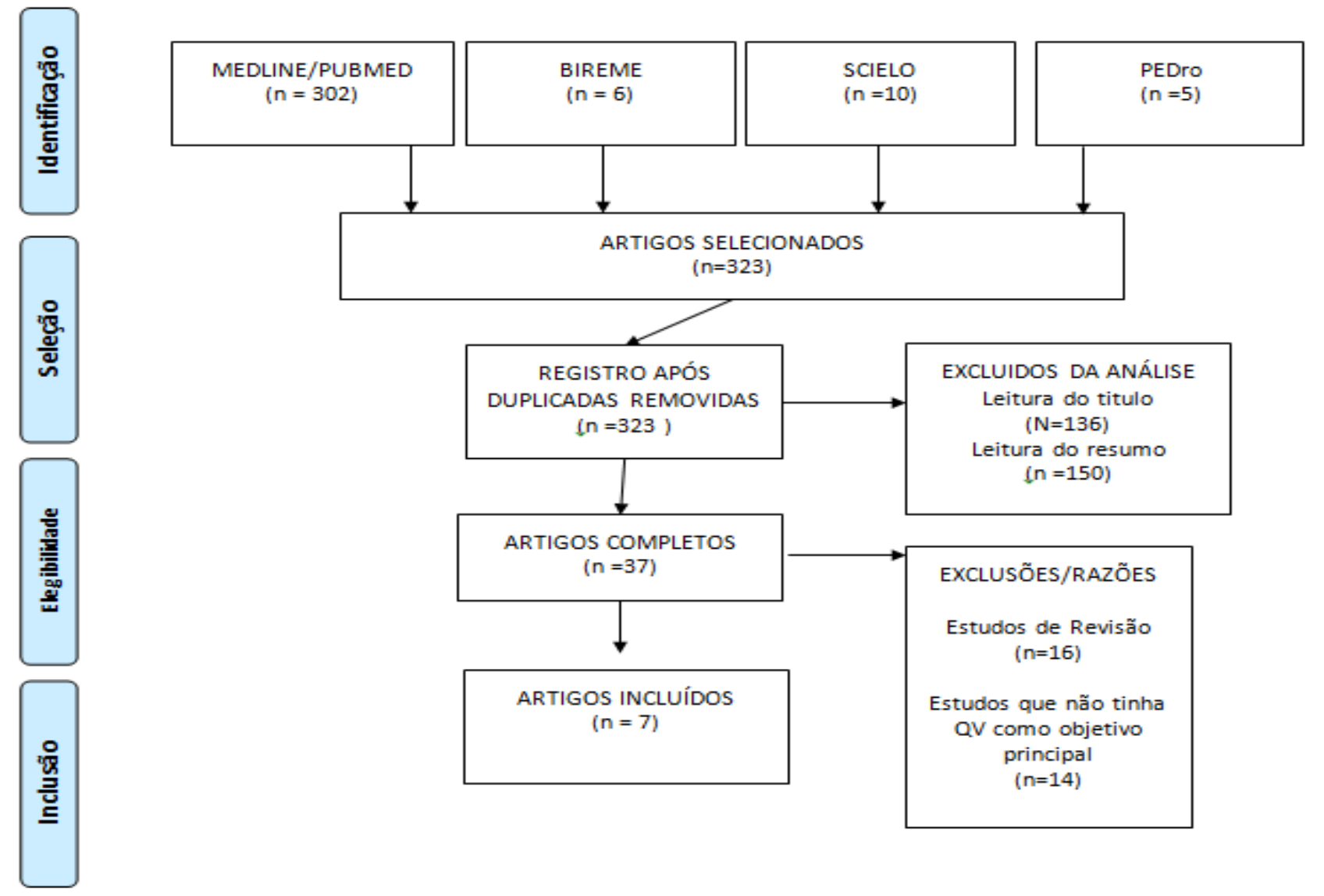

Fonte: Silva APR, et al. (2019).

\section{DISCUSSÃO}

O adoecimento oncológico afeta negativamente tanto a capacidade do paciente de realizar atividades diárias quanto a sua Qualidade de Vida (QV), pois o mesmo passa a viver uma constante incerteza quanto à evolução da sua doença e sua sobrevivência. O diagnóstico precoce e os avanços que estão ocorrendo no tratamento clinico estão permitindo uma alta porcentagem de sobreviventes na população com neoplasias malignas (MOROS MT, et al., 2010).

No entanto, os sobreviventes de câncer podem apresentar uma variedade de efeitos colaterais decorrente do tratamento anti-neoplásico, incluindo dor, fadiga, náuseas, depressão, perda de funcionalidade, entre outros efeitos que estão associados a uma diminuição da percepção de QV. Assim, os recursos de tratamento utilizados como a quimioterapia, radioterapia, hormonioterapia e cirurgia interferem negativamente na qualidade de vida relacionada a saúde (QVRS) devido a ampla gama de efeitos colaterais (ZHOU Y, et al., 2017).

Com o objetivo de minimizar esses efeitos, as intervenções com exercícios físicos demonstraram uma redução significativamente de sintomas para os sobreviventes de câncer havendo maior redução em distúrbios como: a depressão e a fadiga, afetando de forma positiva a percepção da QVRS, bem-estar emocional e capacidade funcional (MOROS MT, et al., 2010). 
As alterações psicológicas e fisiológicas enfrentadas pelos sobreviventes de neoplasias podem ser prevenidas, atenuadas, tratadas e/ou reabilitadas por meio de um programa de exercício físico. Trazendo como mais um ponto positivo para a melhora da QV dessa população e que pode evitar a recorrência da doença ou o aparecimento de outro tipo de câncer. A prática de exercício é um fator ouro para esses indivíduos, em especial, essas pessoas devem ser estimuladas a se engajarem em um programa para melhorar a QVRS (JUNIOR MP e JORDÃO P, 2016).

No estudo de Brown JC, et al. (2018) o treinamento de exercícios aeróbicos de intensidade moderada entre sobreviventes de câncer de colón, trouxe benefícios fisiológicos em vários desfechos de QVRS dentre eles, a função física, QV, qualidade do sono e fadiga. Assim como, houve melhoras também nos componentes do bemestar físico, bem-estar emocional e bem-estar funcional. Estes resultados mostram a importância do exercício para controlar ou atenuar as preocupações relacionadas à recorrência da doença nesta população.

A literatura cientifica apresenta outras evidências que confirmam a relação positiva entre a prática de exercícios físicos na população oncológica e a melhora da percepção de QV por indivíduos submetidos a essa modalidade de tratamento, como no estudo de Zhou Y et al. (2017).

No presente estudo, que consistia em um programa de exercícios aeróbicos de intensidade moderada em casa, com duração de 150 minutos por semana, realizados por seis meses, apresentou como resultados positivos a melhora da QVRS em comparação com o braço controle. Além disso, ainda houve melhora estatisticamente significativa no escore de fadiga apenas para os praticantes de exercício (ZHOU Y et al., 2017).

Os achados anteriores corroboram com o estudo de Shobeiri $F$, et al. (2016), que contou com uma intervenção que consistia em um programa de exercícios aeróbicos de intensidade moderada em casa, com duração de seis meses. As mulheres selecionadas tiveram que participar 150 minutos por semana de exercícios aeróbicos de intensidade moderada como caminhada rápida, sendo recomendado para sobreviventes de câncer pelo Colégio Americano de Medicina esportiva (ACSM - The American College of Sport Medicine) e pela Sociedade Americana de Estudo do Câncer (American Cancer Society).

Houve uma mudança significativa na QVRS física maior no grupo que exercitou 150 ou mais minutos por semana em comparação com aqueles que se exercitaram menos, e houve também uma melhora significativa no escore de fadiga (Avaliação Funcional da Terapia do Câncer- Fadiga) para os participantes do grupo de exercício e não houve diferenças entre os grupos quanto à mudança na QVRS mental (SHOBEIRI F et al., 2016).

Complementando os achados anteriores, pôde ser observados os benefícios do exercício físico aeróbico no estudo de Filha JGLC, et al. (2016), na qual obteve melhorias nos domínios vitalidade, aspectos sociais e limitações por aspectos emocionais, o exercício físico durante o tratamento do câncer ajuda na melhora psicológica, social e física.

Entre as modalidades de exercícios, podem ser observados também os benefícios do treinamento resistido na melhoria de parâmetros funcionais e psicológicos, como visto na pesquisa de Hasgtrom AD, et al. (2015), que apresenta resultados positivos do treinamento resistido na QV e fadiga em sobreviventes de câncer de mama. Os autores reforçam que a prática de exercícios resistidos a essa população trazem melhorias nas alterações psicológicas como as percepções de fadiga e QV, além do aumento força muscular, resistência e consequentemente ganhos na funcionalidade global de forma significativa. Os estudos utilizados nessa revisão convergem no entendimento de que vários domínios como o estado global da saúde, função física, emocional e social, dor, autoestima, condição cardiorrespiratória, depressão e fadiga, podem apresentar melhoras significativas devido a prática de atividade física, e que essa prática pode ser eficaz em diminuir sintomas decorrentes do câncer ou do tipo de tratamento utilizado. Assim como, há um entendimento de que não se exercitar pode trazer prejuízos para estes pacientes. Com isso, o exercício físico pode ser considerado uma importante estratégia para melhorar a saúde (LANDRY S, et al., 2018, PAULO TRS, et al., 2019, SEIXAS RJ, et al., 2010). 


\section{CONSIDERAÇÕES FINAIS}

Há na literatura atual evidência de que pacientes com câncer que aderem a programas de tratamentos com exercícios físicos podem ser beneficiados com a melhora de sua qualidade de vida. No passado, as principais prescrições médicas eram enfatizadas no repouso, já que a fadiga está entre os principais sintomas encontrados, porém, essa orientação mudou decorrente do avanço da pesquisa no campo da atividade física, principalmente voltados para as populações que enfrentam doenças crônicas não transmissíveis, como o câncer. Portanto não desistir de fazer atividade física após o diagnóstico de câncer pode contribuir muito para a melhora da percepção de qualidade de vida dos pacientes e consequentemente o controle de sintomas da doença ou do seu tratamento. Enfatizamos ainda, a necessidade de mais estudos com uma diversidade maior de protocolos de tratamentos com exercícios físicos em populações com os mais diversos tipos de neoplasias malignas, para reforçar os benefícios desse valioso recurso em sobreviventes de câncer.

\section{REFERÊNCIAS}

1. BROWN JC, et al. A Randomized Dose-Response Trial of Aerobic Exercise and Health-Related Quality of Life in Colon Cancer Survivors, USA. Journal of the Psychological, Social and Behavioral Dimensions of Cancer, 2018; 27: 12211228.

2. FILHA JGLC, et al. Influências do Exercício Físico na Qualidade de Vida em Dois Grupos de Pacientes com Câncer de Mama, Porto Alegre. Revista Brasileira de Ciências do Esporte, 2016; 38: 107-114.

3. FRENSHAM LJ, et al. Effect of a 12-Week Online Walking Intervention on Health and Quality of Life in Cancer Survivors: A Quasi-Randomized Controlled Trial, Switzerland. International Journal of Environmental Research and Public Health, 2018; 15: 1-17.

4. GUERRA MR, et al. The risk of cancer in Brazil: tendencies and recent epidemiologic studies, Brasil. Revista Brasileira de Cancerologia, 2005; 51: 227-234.

5. HAGSTROM AD, et al. Resistance training improves fatigue and quality of life in previously sedentary breast cancer survivors: a randomized controlled trial, Austrália. European Journal of Cancer Care, 2015; 25: 784-94.

6. INUMARU LE, et al. Fatores de risco e de proteção para câncer de mama: uma revisão sistemática, Brasil. Cad. Saúde Pública, 2011; 27: 1259-1270.

7. JUNIOR MP, JORDÃO P. Efeito do exercício físico sobre a qualidade de vida e a composição corporal em sobrevivente de câncer de mama: um estudo de caso. Revista Brasileira de Prescrição e Fisiologia do Exercício, 2016; 10(61): 602608.

8. KALIKS RA, et al. Diferenças no tratamento sistêmico do câncer no Brasil: meu SUS é diferente do teu SUS, São Paulo. Revista Braz J Oncol, 2017; 13: 1-12.

9. LEITE FMC, et al. Mulheres com Diagnóstico de Câncer de Mama em Tratamento com Tamoxifeno: Perfil Sociodemográfico e Clínico, Espírito Santo. Revista Brasileira de Cancerologia, 2011; 57: 15-21.

10. LANDRY S, et al. Influence of an Adapted Physical Activity Program on Self-Esteem and Quality of Life of Breast Cancer Patients after Mastectomy, Suiça. Journal Kargera, 2018; 95: 188-191.

11. MAKLUF ASD, et al. Avaliação da qualidade de vida em mulheres com câncer de mama, Minas Gerais. Revista Brasileira de Cancerologia, 2006; 52: 49-58.

12. MOREIRA ECH, MANAIA CAR. Quality of life of patient who undergoing mastectomies assisted by the service of physiotherapy of University Hospital of Londrina State University. Revista Ciências Biológicas e da Saúde, 2005; 26(1): 21-30.

13. MOROS MT, et al. Ejercicio físico en mujeres con cáncer de mama, Santiago. Rev. méd. Chile, 2010; $138: 715-722$.

14. MURTEZANI A, et al. The effect of aerobic exercise on quality of life among breast cancer survivors: a randomized controlled trial, India. Journal of Cancer Research and Therapeutics, 2014; 10: 658-64.

15. PAULO TRS, et al. The impact of an exercise program on quality of life in older breast cancer survivors undergoing aromatase inhibitor therapy: a randomized controlled trial, São Paulo. Rev. Health Qual Life Outcomes, $2019 ; 17: 1$-12. SEIXAS RJ, et al. Atividade Física e Qualidade de Vida em Pacientes Oncológicos durante o Período de Tratamento Quimioterápico.Revista Brasileira de Cancerologia, 2010; 56: 321-330.

16. SHOBEIRI F, et al. The Impact of Aerobic Exercise on Quality of Life in Women with Breast Cancer: A Randomized Controlled Trial, Irã. Journal of Research in Health Sciences, 2016; 16: 127-132.

17. TOLENTINO GP, et al. Breast cancer and physical exercise, Brasil. Revista Brasileira de Medicina, $2016 ; 67: 78$-81.

18. VOSGERAU DSR, ROMANOWSKI JP. Estudos de revisão: implicações conceituais e metodológicas. Revista Diálogo Educ, 2014; 14(41): 165-189.

19. ZHOU Y, et al. Randomized Trial of Exercise on Quality of Life in Women with Ovarian Cancer: Women's Activity and Lifestyle Study in Connecticut (WALC), Boston. Journal of the National cancer Institute, 2017; 109: 1-7. 